\title{
Dendritic cell recruitment in lesions of human and experimental pulmonary hypertension
}

\author{
F. Perros*,\#, , P. Dorfmüller*,\#, , R. Souza*, I. Durand-Gasselin", S. Mussot ${ }^{+}$, \\ M. Mazmanian ${ }^{+}$, P. Hervé ${ }^{*,+}$, D. Emilie ${ }^{\#}$, G. Simonneau* and M. Humbert*,\#
}

ABSTRACT: In the present study, the hypothesis that dendritic cells (DCs), key players in immunity and tolerance, might be involved in the immunopathology of idiopathic pulmonary arterial hypertension (IPAH) was tested.

The phenotype and localisation of DCs were characterised by immunohistochemistry and double-labelling immunofluorescence in lung samples from controls, human IPAH patients and an experimental pulmonary hypertension model (monocrotaline-exposed rats).

As compared with controls, morphometric analysis demonstrated increased numbers of dendritic cell-specific intercellular adhesion molecule-grabbing nonintegrin (DC-SIGN)-positive cells in muscular pulmonary arteries in IPAH and OX-62-positive DCs in monocrotaline-induced pulmonary hypertension. In human samples, the mean $\pm S E M$ number of DC-SIGN-positive cells artery $^{-1}$ of $100-300 \mu \mathrm{m}$ diameter was $1.4 \pm 0.4$ in controls versus $26.4 \pm 2.7$ in IPAH. In rats, the number of OX-62-positive cells artery $^{-1}$ of $50-150 \mu \mathrm{m}$ diameter was $0.5 \pm 0.2$ in controls, and $0.7 \pm 0.5,3.1 \pm 0.5$ and $8.4 \pm 0.6$ at day 7,14 and 28 after monocrotaline exposure, respectively. Human complex lesions of muscular pulmonary arteries showed transmural DC infiltration. Phenotyping revealed an immature DC profile in human and experimental pulmonary hypertension.

The results support the concept that immature dendritic cells accumulate in remodelled pulmonary vessels and hence could be involved in the immunopathology of pulmonary hypertension.

KEYWORDS: Dendritic cells, immunopathology, inflammation, monocrotaline, pulmonary arterial hypertension
( nflammatory mechanisms play a role in pulmonary arterial hypertension (PAH) [1]. Indeed, severe PAH may complicate the course of systemic inflammatory conditions, and treatment with corticosteroids and immunosuppressants has been shown to improve PAH in patients with connective tissue diseases such as systemic lupus erythematosus [2, 3]. In addition, pathogenic autoantibodies targeting endothelial cells are capable of inducing vascular endothelial apoptosis and may initiate the development of PAH $[4,5]$. A subset of idiopathic PAH (IPAH) patients has been shown to have circulating autoantibodies (antinuclear, antiendothelial and antifibroblast antibodies) $[6,7]$ as well as elevated circulating levels of the pro-inflammatory cytokines interleukin (IL)-1 and IL-6 [8]. Lung histology of patients displaying severe IPAH

frequently reveals inflammatory cell infiltrates corresponding to macrophages and lymphocytes in the range of plexiform lesions with local expression of chemokines such as RANTES (regulated on activation, normal T-cell expressed and secreted) and fractalkine [9, 10]. Experimental data from monocrotaline-induced pulmonary hypertension in rats further support a link between pulmonary vascular inflammation and remodelling.

Among the different pathways related to the inflammatory process, the role of dendritic cells (DCs) has been increasingly recognised, not only as simple antigen-presenting cells responsible for the initiation of the inflammatory response, but, especially, as a key modulator of the whole process, thus raising its importance in many

For editorial comments see page $\mathbf{4 3 5}$.

The present publication reflects only the views of the author(s) and the European Community is in no way liable for any use that may be made of the information contained therein.
AFFILIATIONS

*UPRES EA2705, Service de Pneumologie, Centre National de Référence de l'Hypertension Artérielle Pulmonaire, Hôpital Antoine-Béclère, Assistance Publique-Hôpitaux de Paris, Université Paris-Sud 11, and

\# Institut National de la Santé et de la Recherche Médicale, U764, Institut Fédératif de Recherche 13, Clamart, +UPRES EA2705, Laboratoire de Chirurgie Expérimentale, Centre Chirurgical Marie Lannelongue, Université Paris-Sud 11, Le Plessis Robinson, France.

'F. Perros and P. Dorfmüller contributed equally to this study.

CORRESPONDENCE

M. Humbert: Service de Pneumologie et Réanimation Respiratoire, Hôpital Antoine-Béclère, 157 rue de la Porte de Trivaux, 92140 Clamart, France. Fax: 33146303824

E-mail: marc.humbert@abc.aphp.fr

Received:

July 192006

Accepted after revision:

November 132006

SUPPORT STATEMENT

F. Perros is supported by a grant from Ministère de la Recherche

(Paris, France). R. Souza is supported by a grant from the ERS. The South Paris Pulmonary Hypertension Centre for Research and Care (Paris) is supported in part by grants from Chancellerie des Universités, Legs Poix, Université Paris-Sud 11 (Paris) and Institut des Maladies Rares (Paris). The present research project received financial support from the European

Commission under FP6 (Contract No: LSHM-CT-2005-018725,

PULMOTENSION)

STATEMENT OF INTEREST

None declared. 
human disorders, including allergy [11], autoimmunity [12], tumour immunology [13] and allograft rejection [14]. Numerous data clearly demonstrate that pulmonary DCs are at least involved in the pathogenesis of a whole spectrum of highly prevalent respiratory conditions, and that for some of those (such as asthma), DCs have been proven to be essential in the development of the disease [15]. In mice, pulmonary DCs have been shown to be the key cells in the pathogenesis of asthma [16] and there is circumstantial evidence from animal models that pulmonary DCs might contribute to the development of chronic obstructive pulmonary disease in smokers [17]. Furthermore, the number of lung DCs is increased in sarcoidosis [18] and diffuse panbronchiolitis [19]. The ability of DCs to differentiate into other cell phenotypes, including endothelial cells [20], expands their potential role in vascular disorders. In the present study, data supporting the involvement of DCs in human and experimental pulmonary hypertension are reported for the first time.

\section{METHODS}

\section{Subjects and sample processing}

Human PAH pulmonary specimens were obtained at the time of lung transplantation in eight patients displaying severe IPAH. Right-heart catheterisation demonstrated severe precapillary pulmonary hypertension in all cases. Control pulmonary specimens consisted of eight lung biopsies performed during surgery for pneumothoraces or at a distance from localised pulmonary lesions. Controls had no evidence of pulmonary vascular disease. $\mathrm{PAH}$ and control lungs were distended by infusion of ornithyl carbamyl transferase compound (OCT; VWR International, West Chester, PA, USA) diluted in PBS (1:5) into the main bronchi or by needle injection into the parenchyma to preserve lung morphology. Specimens for histology were collected from lung periphery, then snap-frozen in isopentane on dry ice and stored at $-80^{\circ} \mathrm{C}$ until further analysis was required. All patients and controls studied were part of the French Network on Pulmonary Hypertension, a programme approved by the institutional Ethics Committee, and gave written informed consent.

\section{Animal model}

Adult male Wistar rats (8-10 weeks old) were euthanised by overdose of sodium pentobarbital 7, 14 and 28 days after a single subcutaneous injection of saline $(\mathrm{n}=10)$ or $60 \mathrm{mg} \cdot \mathrm{kg}^{-1}$ monocrotaline (Sigma-Aldrich, St Louis, MO, USA; $n=30$ ). Lungs from controls (saline) and monocrotaline-exposed rats were distended by infusion of OCT:PBS (1:1) into the trachea, then snap-frozen. Monocrotaline-exposed rats displayed severe pulmonary hypertension at day 28, while mild haemodynamic and histological alterations occurred at day 14. Saline-exposed rats and rats evaluated 7 days after monocrotaline exposure had normal pulmonary haemodynamic values and pulmonary vascular histology. Animal experiments were approved by the administrative panel on animal care at Centre de Chirurgie Expérimentale Marie Lannelongue, Le Plessis-Robinson, France.

\section{Immunohistochemistry}

Immunohistochemistry was performed on $7-\mu \mathrm{m}$ sections of frozen lung tissue. After routine preparation and peroxydase inhibition, human samples were processed with the following antibodies: monoclonal antibodies against dendritic cell-specific intercellular adhesion molecule-grabbing nonintegrin (DCSIGN; Pharmingen clone DCN46; BD Pharmingen, San Diego, CA, USA), CD1a (Pharmingen clone HI149; Pharmingen) and CD83 (Immunotech clone HB15A; Immunotech, Mississauga, ON, Canada) diluted in PBS containing $1 \%$ calf foetal serum and $5 \%$ human $\mathrm{AB}$ serum. Rat DCs were stained with the monoclonal anti-CD103 antibody (Pharmingen clone OX-62; Pharmingen) diluted in PBS containing $2 \%$ normal rat serum. After overnight incubation at $4^{\circ} \mathrm{C}$, human lung sections were labelled with biotinylated anti-mouse immunoglobulins and peroxidase-labelled streptavidin (Biogenex, San Ramon, CA, USA). Rat OX-62 labelling was revealed with the labelled streptavidin-biotin (LSAB)2 kit for use on rat specimens (Dako A/S, Glostrup, Denmark). Staining was completed after incubation with substrate-chromogen (3-amino-9-ethylcarbazole) solution (Dako A/S). Slides were counterstained with Mayer's hematoxylin (Réactifs RAL, Martillac, France) and mounted with aqueous medium (Glycergel; Dako A/S). Controls used for these antibodies included omission of the primary antibody and substitution of the primary antibody by isotype control. Positive control for CD1a and CD83 was obtained by staining hilar lymph nodes of explanted lungs (data not shown).

\section{Immunofluorescent labelling}

For double immunofluorescence, antihuman DC-SIGN (Pharmingen clone DCN46; Pharmingen) and OX-62 (Pharmingen) antibodies were labelled by biotinylated anti-mouse immunoglobulins and streptavidin (Biogenex or Dako A/S), and AlexaFluor 594 or 488 conjugate (Molecular Probes, Paisley, UK) for $1 \mathrm{~h}$. The tissues were subsequently incubated overnight either with fluorescein isothiocyanate conjugated, anti-smooth muscle $\alpha$-actin (Sigma Aldrich clone 1A4, Sigma Aldrich), antihuman CD68 (Dako clone KP1, Dako A/S), anti-rat RTIB (HMC class II, Serotec clone OX6; Serotec, Oxford, UK), rhodamine-conjugated vimentin (Santa Cruz sc-6260, Santa Cruz Biotechnology Inc., Santa Cruz, CA, USA), or Alexa Fluor 488 conjugated anti-rat CD68 (Serotec clone ED1; Serotec) and anti-rat CD4 (Serotec clone W3/25; Serotec). The slides were mounted with Vectashield Mounting Medium (Vector Laboratories, Burlingame, CA, USA).

\section{Immunohistochemical cell quantification}

Immunohistochemical cell quantification was carried out using a Nikon $80 \mathrm{i}$ microscope with the morphometric analysis software NSI Elements (Nikon, Tokyo, Japan). The mean number of pulmonary arterial DCs was calculated by counting DC-SIGN (human DCs) or OX-62 (rat DCs) expressing cells on three slides of each sample. For quantification, pulmonary muscular arteries with a cross-sectional diameter of 100-300 or 50-150 $\mu \mathrm{m}$ for human and rat lungs, respectively, were taken into account.

\section{Statistical analysis}

All values are expressed as mean \pm SEM. In human samples, statistical significance was evaluated with a two-tailed unpaired t-test for comparisons between control and PAH groups. ANOVA using repeated measures and the Fisher projected least significant difference (PLSD) post test was performed on rat results (4 groups: control, day 7,14 and 28 after monocrotaline exposure). A p-value $<0.05$ was considered significant. 


\section{RESULTS}

\section{Detection of dentritic cells in pulmonary arteries from IPAH} patients

In all human lung samples from IPAH patients, DC-SIGNexpressing DCs presenting network-like organisation were identified in adventitial connective tissue of pulmonary vasculature (fig. 1). Affected vessels in PAH lungs displayed DC infiltration into the adventitial and the medial layer. Transmural infiltration by DCs was frequently observed in plexiform lesions. Morphometric analysis revealed a dramatic increase in the number of DC-SIGN-positive cells in muscular pulmonary arteries: the mean number of DC-SIGNpositive cells artery $^{-1}$ of $100-300 \mu \mathrm{m}$ diameter was $1.4 \pm 0.4$ in controls versus $26.4 \pm 2.7$ in IPAH $(p<0.001$, two-tailed unpaired t-test; fig. 2). Immunohistochemistry indicated that these cells were CD1a- and CD83-negative, corresponding to immature DCs (data not shown). Immunofluorescent double labelling with CD68, $\alpha$-actin and vimentin excluded possible cross-reactions with macrophages or fibroblasts (fig. 1). Pulmonary arteries of control lungs showed scattered adventitial DC staining. To a smaller extent, DC-SIGN-positive cells were detected in peribronchial lung parenchyma, lobular septa and visceral pleura of lungs from both IPAH patients and controls. DC-SIGN-positive cells were not found in venules or capillaries.

\section{Detection of dentritic cells in pulmonary arteries from experimental pulmonary hypertension}

The recruitment of DCs in monocrotaline-induced pulmonary hypertension was studied in Wistar rats (fig. 3). DCs were detected by the CD103 clone OX-62, which has been shown to be an important marker of rat DCs [21]. An increase in the number of DCs was first observed 14 days after monocrotaline exposure. By 28 days after monocrotaline injection, pulmonary hypertensive rats had a marked recruitment of OX-62-positive DCs in the adventitial layer of muscular pulmonary arteries, as well as in the vessel wall of pulmonary veins (fig. 2). The mean number of OX-62-positive cells artery $^{-1}$ of 50-150 $\mu$ m diameter was $0.5 \pm 0.2$ in controls, and $0.7 \pm 0.5,3.1 \pm 0.5$ and $8.4 \pm 0.6$ at day 7,14 and 28 after monocrotaline exposure, respectively $(p<0.001$, ANOVA; fig. 2). DC counts increased with the degree of pulmonary artery medial remodelling and were low in control vessels. Immunofluorescence double-labelling revealed an immature pattern of the recruited DCs: CD4negative; OX-62-positive; CD68-negative; mostly major histocompatibility complex class II-negative phenotype (fig. 3). OX62-positive scattered DCs were present in bronchial-associated lymphatic tissue, as well as in the bronchial wall of PAH rats and controls.

\section{DISCUSSION}

The present study reports the first evidence that DCs may contribute to the immunopathology of human and experimental pulmonary hypertension. The results suggest that in human and experimental pulmonary hypertension, immature DCs accumulate in remodelled pulmonary vessels and hence could be involved in the evolution of the pulmonary vascular lesions. The present study has shown that mean numbers of arterial DCs increase during the development of vasculopathy in monocrotaline-exposed rats. The arterial DC accumulation
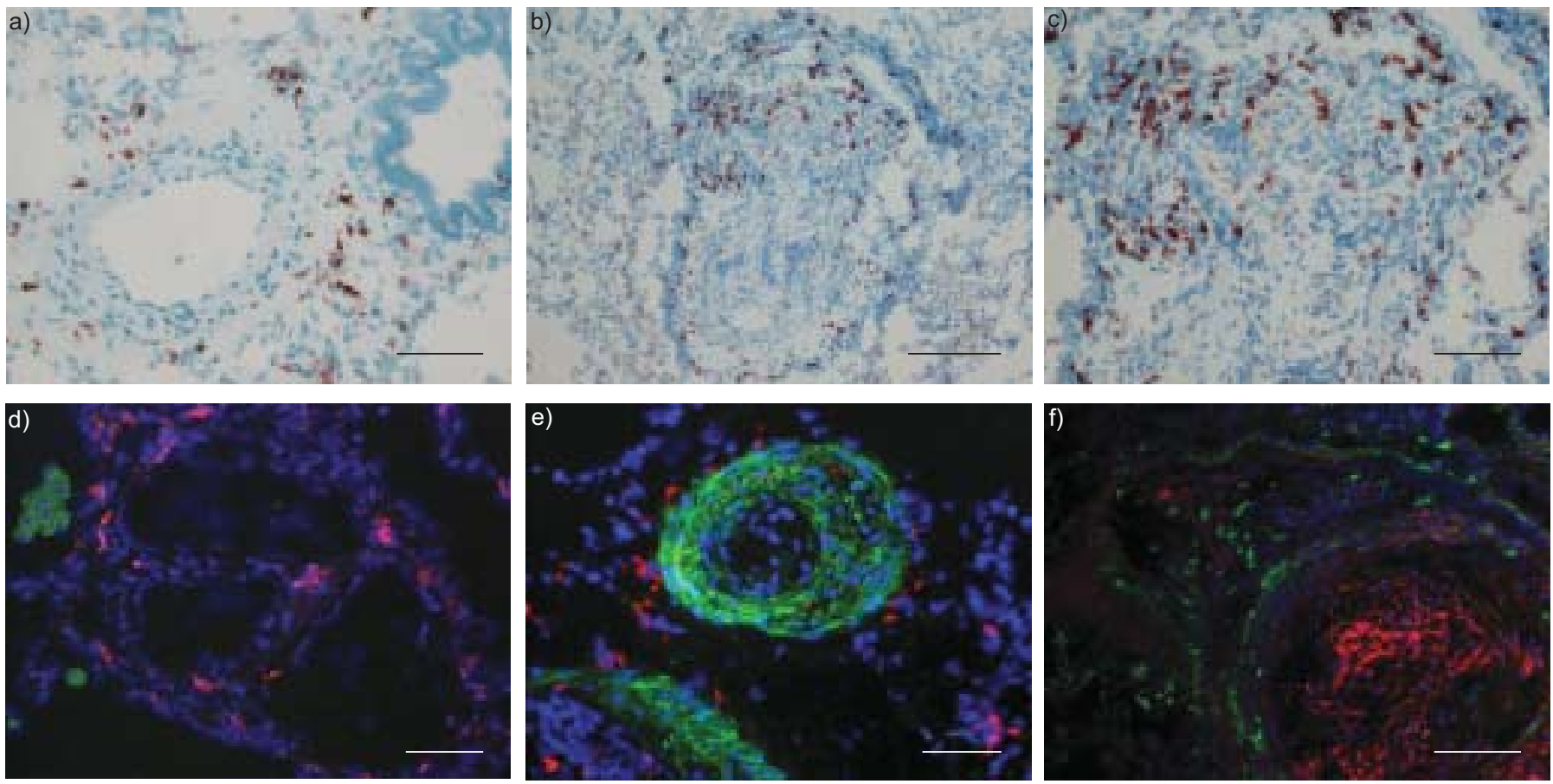

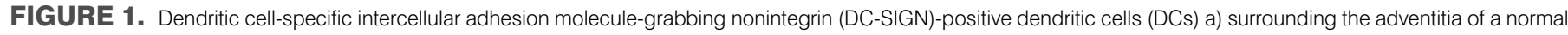

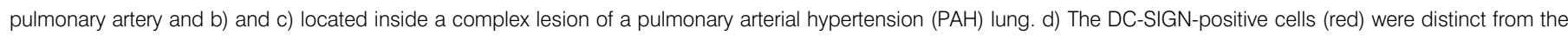

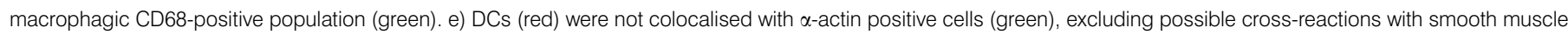

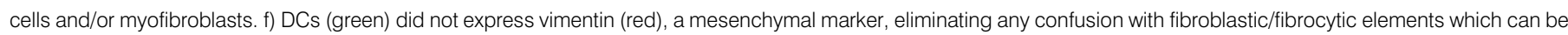
involved in fibrotic PAH lesions. Scale bars $=100 \mu \mathrm{m}$, except b), scale bar $=200 \mu \mathrm{m}$. 

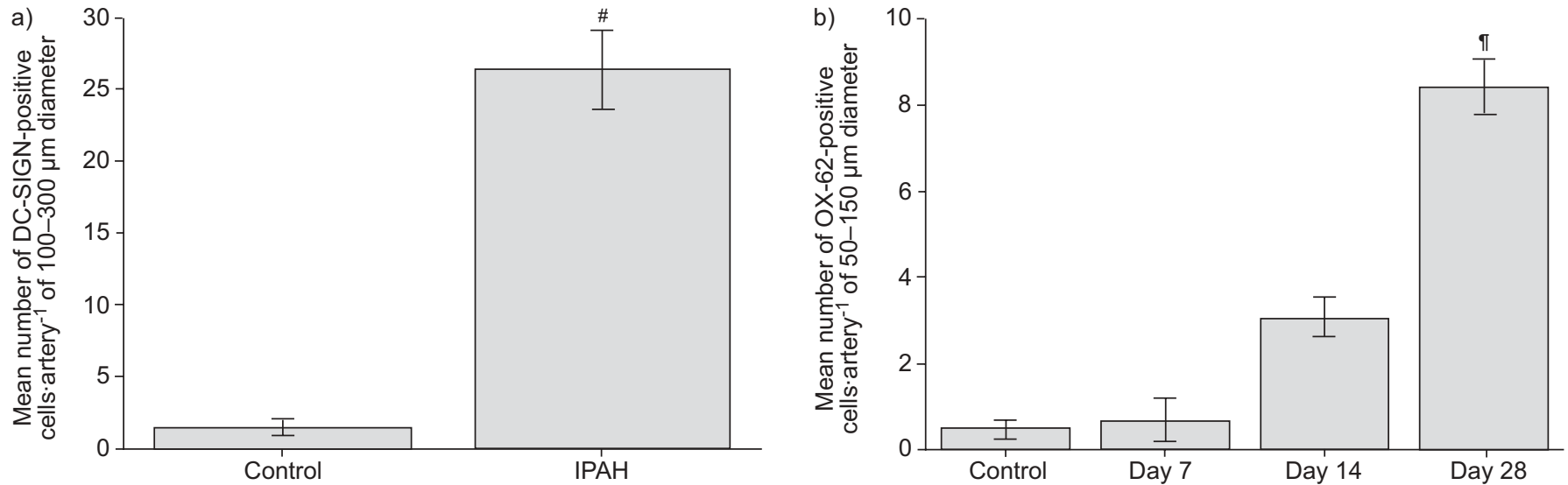

FIGURE 2. Quantification of dendritic cells in human and rat pulmonary arteries. a) In idiopathic pulmonary arterial hypertension (IPAH) patients, an increase in the number of dendritic cell-specific intercellular adhesion molecule-grabbing nonintegrin (DC-SIGN)-positive cells in pulmonary arteries of 100-300 $\mu \mathrm{m}$ diameter is observed. b) Monocrotaline-exposed rats show a significant increase in the mean number of OX-62-positive cells in pulmonary arteries of 50-150 $\mu \mathrm{m}$ diameter, in a time-related fashion. ${ }^{*}$ : $p<0.001$, two-tailed unpaired t-test; ${ }^{\top}: p<0.001$, ANOVA.

precedes pulmonary arterial thickening and haemodynamic alteration and is constantly present in remodelled vessels, indicating that DC influx is not merely a consequence of increased pulmonary arterial pressure. Functional testing of DC recruitment, such as in vivo migration in the animal model, has not been performed in the present study. Therefore, DC accumulation in lesions of pulmonary hypertension may be the consequence of recruitment but also of local proliferation, decreased efflux from the pulmonary vascular wall or even transdifferentiation from infiltrating macrophages.

The role of inflammatory processes in the natural history of PAH has been discussed since inflammatory infiltrates surrounding remodelled vessels in human $\mathrm{PAH}$ have been described by several investigators. In addition, increased levels and expression of cytokines in blood, lung parenchyma and
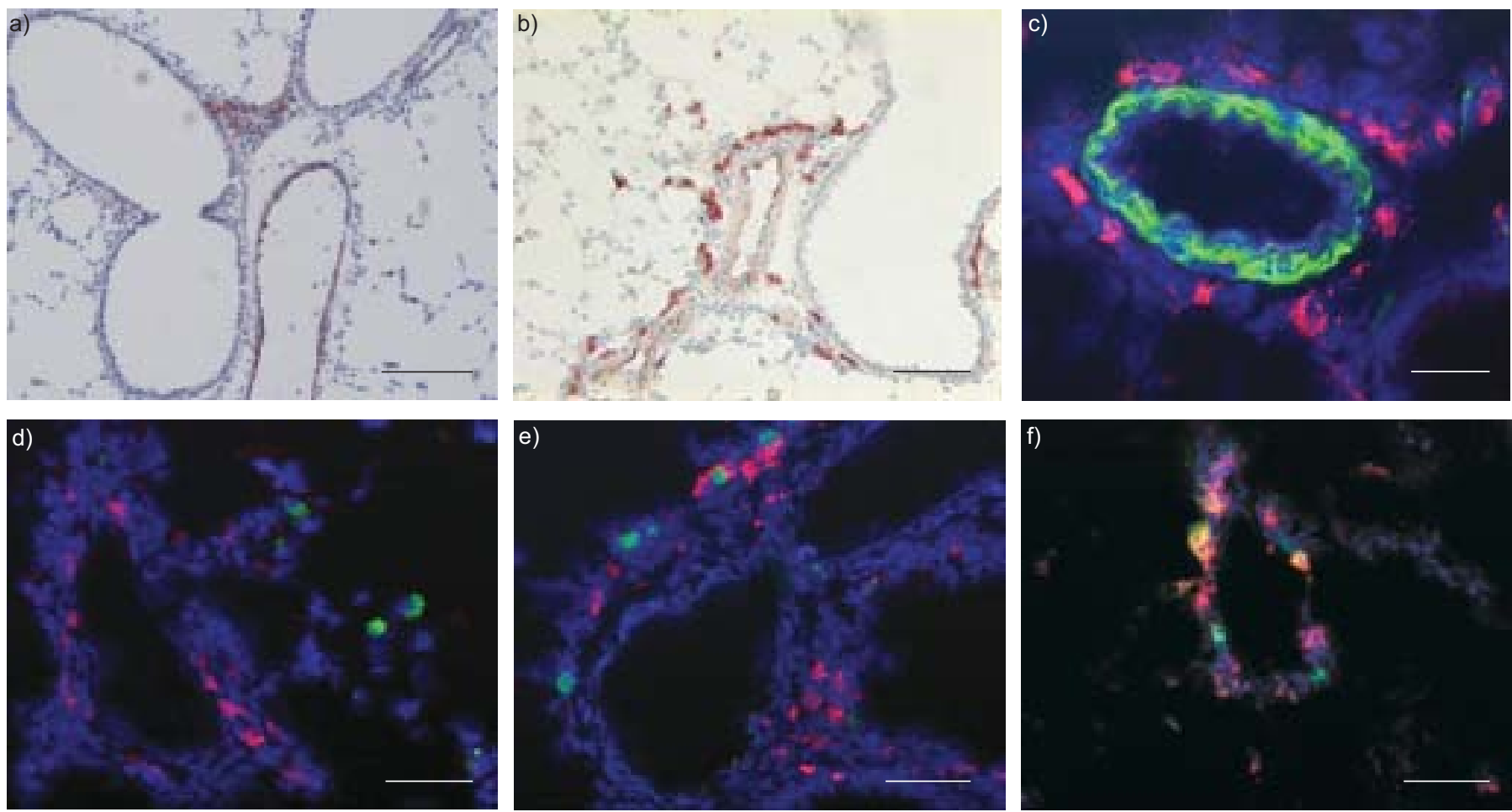

FIGURE 3. OX-62-positive rat dendritic cells (DCs) were detected only a) in bronchus-associated lymphatic tissue of control lungs, while b) presenting a network-like organisation in the adventitia of affected arteries in monocrotaline-exposed rats. Recruited OX-62-positive DCs (red) were distinct from the c) medial smooth muscle cells (green), and also from d) macrophagic populations (green), e) rat monocytic/lymphocytic cells and DC CD4-positive subsets (green). f) OX-62 (red) and major histocompatibility complex class II (green) were occasionally coexpressed (yellow staining), highlighting different maturation stages or different DC subpopulations. a) Scale bar $=200 \mu \mathrm{m}$, b) scale bar $=100 \mu \mathrm{m}, \mathrm{c}-\mathrm{f}$ ) scale bars $=25 \mu \mathrm{m}$. 
affected pulmonary arteries have been described. The increased prevalence of $\mathrm{PAH}$ in connective tissue disorders and, in some forms, its successful treatment with immunosuppressive therapy, support the concept of inflammatory pathways in the development and modulation of the disease. Therefore, a better understanding of the relationship between inflammation and pulmonary vascular remodelling is of major importance.

Inflammatory mechanisms and DCs could play a role in the early stages of human PAH development, as demonstrated in the rat inflammatory model of monocrotaline-induced pulmonary hypertension. All human PAH samples in the present study came from patients suffering from IPAH. This study design was chosen in order to avoid associated inflammatory conditions which can be encountered in PAH related to connective tissue diseases, autoimmune thyroiditis or HIV infection. The goal was to confront an exaggerated inflammatory animal model of pulmonary hypertension (monocrotaline-induced pulmonary hypertension) to "pure" IPAH and to describe common inflammatory mechanisms. Indeed, such mechanisms have not yet been well defined in human IPAH.

As has been shown previously, human lung DCs lack CD83 expression [22], corresponding to immature DCs [23]. Also, CD1a, a characteristic cell-surface molecule present on epidermal and blood-derived DCs was consistently absent on perivascular lung DCs [22]. CD1a was occasionally seen in bronchial epithelium (data not shown), as confirmed by DEMEDTS et al. [24]. This observed lack of mature DCs may be explained by the fact that after processing foreign/self antigens, DCs subsequently migrate to lymphoid tissues, where maturation is achieved. Here, DCs initiate the activation of antigen-specific T-cells. Accordingly, rat lung DCs presented the immature CD4-negative OX-62 positive phenotype [25]. Fibrocytes comprise a subpopulation of circulating mononuclear cells of a monocyte/macrophage CD68-positive lineage that can exhibit fibroblast properties at the site of tissue injury ( $\alpha$-actin and vimentin production, differentiation into myofibroblasts) [26, 27], which were shown to participate in the arterial remodelling that occurs in hypoxiainduced PAH in animal models [26]. The current authors checked, by immunofluorescent double-labelling, that human DC-SIGN- and rat OX-62-positive cells did not express vimentin or $\alpha$-actin fibrocytic markers. Hence, it can be concluded that a DC subpopulation distinct from fibroblast/ fibrocyte lineages accumulates in human and rat remodelled pulmonary arteries.

Among the different lineage of inflammatory cells, DCs have been linked to vascular disorders. In systemic arteries, vascular-associated DCs densely accumulate in arterial regions that are under major haemodynamic stress via turbulent flow conditions [28]. Interestingly, branching points of pulmonary arteries with altered flow conditions are predisposed to the development of plexiform lesions in human PAH [29]. Of note, the current authors observed that transmural infiltration by DCs was most frequently observed in plexiform lesions. Concomitantly to the haemodynamic changes, occurrence of oxidative stress in PAH could act as a DC-attracting "danger signal". Indeed, it has been shown that markers of oxidative stress are constantly present in endothelial and medial layers of pulmonary arteries in PAH [30].

DC-SIGN is a C-type lectin which is highly expressed on the surface of immature DCs [31] and has been shown to allow monocyte-derived DCs to recognise a variety of microorganisms including viruses, parasites and bacteria [32]. DCSIGN can also bind adhesion molecules on the surface of naive $\mathrm{T}$-cells and endothelium suggesting its involvement in T-cell activation and DC trafficking [31]. It is noteworthy that recent findings highlight the involvement of a subset of DCs in tumour angiogenesis, by undergoing endothelial-like differentiation ex vivo and assembling neovasculature in vivo [20].

Novel therapeutic strategies may target pulmonary artery remodelling and its inflammatory component in PAH. SCHERMULY et al. [33] have reported that the platelet-derived growth factor receptor antagonist STI571 (imatinib mesylate) reverses pulmonary vascular remodelling in two different animal models of pulmonary hypertension, including monocrotaline-exposed rats. Moreover, preliminary case reports provide evidence in support of imatinib as an antiremodelling agent in severe human IPAH [34-36]. Although the curative properties of imatinib in experimental and human PAH are supposed to rely on in vitro-demonstrated antiproliferative, antimigratory and pro-apototic effects on pulmonary artery smooth muscle cells [33], recent publications highlight the DC-modulatory effects of imatinib in vitro and in vivo [37-40]. Indeed, MOHTY et al. [39] have shown that disease response to imatinib in patients with chronic myeloid leukemia (CML) is accompanied by restoration of plasmacytoid DCs function in vivo. These findings provide evidence that imatinib is capable of restoring some DC-related immune functions in CML. In addition, SMYTH [40] states that imatinib can activate a cell type named "interferon-producing killer DCs", considered to have characteristics of both DCs and natural killer cells. Moreover, a recent report indicates that imatinib activates interferon-producing killer DCs in a mouse model of cancer, explaining the therapeutic effect of this drug in some gastrointestinal stromal tumours which are resistant to its antiproliferative effect [41]. Thus, imatinib effects in IPAH could be linked at least in part to the modulation of DC function.

In conclusion, the possible contribution of dendritic cells to the immunopathology of both human and experimental pulmonary hypertension is of major interest. Further phenotyping of the immature dendritic cell-specific intercellular adhesion molecule-grabbing nonintegrin-positive subsets in lungs of human pulmonary arterial hypertension should help to better characterise dendritic cells in pulmonary arterial hypertension. The possible involvement of immature dendritic cells in a wellestablished animal model of inflammatory pulmonary hypertension will aid the analysis of the effects of specific therapeutic interventions to modulate the role of these cells in vivo. Finally, evaluation of the cross-talk between immature dendritic cells and endothelial and smooth muscle cells should be evaluated in vivo and in vitro. These data will allow the better understanding of the links between immune cells, abnormal angiogenesis and pulmonary artery remodelling in pulmonary hypertension. 


\section{REFERENCES}

1 Dorfmüller $\mathrm{P}$, Perros $\mathrm{F}$, Balabanian $\mathrm{K}$, Humbert $\mathrm{M}$. Inflammation in pulmonary arterial hypertension. Eur Respir J 2003; 22: 358-363.

2 Montani D, Achouh L, Marcelin AG, et al. Reversibility of pulmonary arterial hypertension in $\mathrm{HIV} / \mathrm{HHV} 8$-associated Castleman's disease. Eur Respir J 2005; 26: 969-972.

3 Sanchez O, Sitbon O, Jais X, Simonneau G, Humbert M. Immunosuppressive therapy in connective tissue diseasesassociated pulmonary arterial hypertension. Chest 2006; 130: 182-189.

4 Nicolls MR, Taraseviciene-Stewart L, Rai PR, Badesch DB, Voelkel NF. Autoimmunity and pulmonary hypertension: a perspective. Eur Respir J 2005; 26: 1110-1118.

5 Mouthon L, Guillevin L, Humbert M. Pulmonary arterial hypertension: an autoimmune disease? Eur Respir J 2005; 26: 986-988.

6 Tamby MC, Chanseaud Y, Humbert M, et al. Antiendothelial cell antibodies in idiopathic and systemic sclerosis associated pulmonary arterial hypertension. Thorax 2005; 60: 765-772.

7 Tamby MC, Humbert M, Guilpain P, et al. Antibodies to fibroblasts in idiopathic and scleroderma-associated pulmonary hypertension. Eur Respir J 2006; 28: 799-807.

8 Humbert M, Monti G, Brenot F, et al. Increased interleukin1 and interleukin-6 serum concentrations in severe primary pulmonary hypertension. Am J Respir Crit Care Med 1995; 151: 1628-1631.

9 Balabanian K, Foussat A, Dorfmuller $\mathrm{P}$, et al. $\mathrm{CX}_{3} \mathrm{C}$ chemokine fractalkine in pulmonary arterial hypertension. Am J Respir Crit Care Med 2002; 165: 1419-1425.

10 Dorfmüller $\mathrm{P}$, Zarka V, Durand-Gasselin I, et al. Chemokine RANTES in severe pulmonary arterial hypertension. Am J Respir Crit Care Med 2002; 165: 534-539.

11 van Rijt LS, Lambrecht BN. Dendritic cells in asthma: a function beyond sensitization. Clin Exp Allergy 2005; 35: 1125-1134.

12 Palucka AK, Blanck JP, Bennett L, Pascual V, Banchereau J. Cross-regulation of TNF and IFN- $\alpha$ in autoimmune diseases. Proc Natl Acad Sci U S A 2005; 102: 3372-3377.

13 Gabrilovich D, Ishida $\mathrm{T}$, Oyama $\mathrm{T}$, et al. Vascular endothelial growth factor inhibits the development of dendritic cells and dramatically affects the differentiation of multiple hematopoietic lineages in vivo. Blood 1998; 92: 4150-4166.

14 Morelli AE, Thomson AW. Role of dendritic cells in the immune response against allografts. Curr Opin Nephrol Hypertens 2000; 9: 607-613.

15 Ardavin C, Amigorena S, Reis e Sousa C. Dendritic cells: immunobiology and cancer immunotherapy. Immunity 2004; 20: 17-23.

16 Lambrecht BN, Hammad H. Taking our breath away: dendritic cells in the pathogenesis of asthma. Nat Rev Immunol 2003; 3: 994-1003.

17 D'Hulst AI, Maes T, Bracke KR, et al. Cigarette smokeinduced pulmonary emphysema in scid-mice. Is the acquired immune system required? Respir Res 2005; 6: 147.

18 Ota M, Amakawa R, Uehira K, et al. Involvement of dendritic cells in sarcoidosis. Thorax 2004; 59: 408-413.
19 Todate A, Chida K, Suda T, et al. Increased numbers of dendritic cells in the bronchiolar tissues of diffuse panbronchiolitis. Am J Respir Crit Care Med 2000; 162: 148-153.

20 Conejo-Garcia JR, Benencia F, Courreges MC, et al. Tumorinfiltrating dendritic cell precursors recruited by a $\beta$ defensin contribute to vasculogenesis under the influence of Vegf-A. Nat Med 2004; 10: 950-958.

21 Brenan M, Puklavec M. The MRC OX-62 antigen: a useful marker in the purification of rat veiled cells with the biochemical properties of an integrin. J Exp Med 1992; 175: 1457-1465.

22 Cochand L, Isler P, Songeon F, Nicod LP. Human lung dendritic cells have an immature phenotype with efficient mannose receptors. Am J Respir Cell Mol Biol 1999; 21: 547-554.

23 Reddy A, Sapp M, Feldman M, Subklewe M, Bhardwaj N. A monocyte conditioned medium is more effective than defined cytokines in mediating the terminal maturation of human dendritic cells. Blood 1997; 90: 3640-3646.

24 Demedts IK, Brusselle GG, Vermaelen KY, Pauwels RA. Identification and characterization of human pulmonary dendritic cells. Am J Respir Cell Mol Biol 2005; 32: 177-184.

25 Trinite B, Chauvin C, Peche H, Voisine C, Heslan M, Josien R. Immature $\mathrm{CD} 4^{-} \mathrm{CD} 103^{+}$rat dendritic cells induce rapid caspase-independent apoptosis-like cell death in various tumor and nontumor cells and phagocytose their victims. J Immunol 2005; 175: 2408-2417.

26 Frid MG, Brunetti JA, Burke DL, et al. Hypoxia-induced pulmonary vascular remodeling requires recruitment of circulating mesenchymal precursors of a monocyte/ macrophage lineage. Am J Pathol 2006; 168: 659-669.

27 Quan TE, Cowper S, Wu SP, Bockenstedt LK, Bucala R. Circulating fibrocytes: collagen-secreting cells of the peripheral blood. Int J Biochem Cell Biol 2004; 36: 598-606.

28 Millonig G, Niederegger H, Rabl W, et al. Network of vascular-associated dendritic cells in intima of healthy young individuals. Arterioscler Thromb Vasc Biol 2001; 21: 503-508.

29 Pietra GG, Capron F, Stewart S, et al. Pathologic assessment of vasculopathies in pulmonary hypertension. J Am Coll Cardiol 2004; 43: Suppl. S, 25S-32S.

30 Bowers R, Cool C, Murphy RC, et al. Oxidative stress in severe pulmonary hypertension. Am J Respir Crit Care Med 2004; 169: 764-769.

31 Soilleux EJ, Morris LS, Leslie G, et al. Constitutive and induced expression of DC-SIGN on dendritic cell and macrophage subpopulations in situ and in vitro. J Leukoc Biol 2002; 71: 445-457.

32 Cambi A, Koopman M, Figdor CG. How C-type lectins detect pathogens. Cell Microbiol 2005; 7: 481-488.

33 Schermuly RT, Dony E, Ghofrani HA, et al. Reversal of experimental pulmonary hypertension by PDGF inhibition. J Clin Invest 2005; 115: 2811-2821.

34 Souza R, Sitbon O, Parent F, Simonneau G, Humbert M. Long term imatinib treatment in pulmonary arterial hypertension. Thorax 2006; 61: 736.

35 Patterson KC, Weissmann A, Ahmadi T, Farber HW. Imatinib mesylate in the treatment of refractory idiopathic 
pulmonary arterial hypertension. Ann Intern Med 2006; 145: 152-153.

36 Ghofrani HA, Seeger W, Grimminger F. Imatinib for the treatment of pulmonary arterial hypertension. $N$ Engl $J$ Med 2005; 353: 1412-1413.

37 Appel S, Balabanov S, Brummendorf TH, Brossart P. Effects of imatinib on normal hematopoiesis and immune activation. Stem Cells 2005; 23: 1082-1088.

38 Appel S, Boehmler AM, Grunebach F, et al. Imatinib mesylate affects the development and function of dendritic cells generated from $\mathrm{CD} 34^{+}$peripheral blood progenitor cells. Blood 2004; 103: 538-544.

39 Mohty M, Jourdan E, Mami NB, et al. Imatinib and plasmacytoid dendritic cell function in patients with chronic myeloid leukemia. Blood 2004; 103: 4666-4668.

40 Smyth MJ. Imatinib mesylate - uncovering a fast track to adaptive immunity. $N$ Engl J Med 2006; 354: 2282-2284.

41 Taieb J, Chaput N, Menard C, et al. A novel dendritic cell subset involved in tumor immunosurveillance. Nat Med 2006; 12: 214-219. 